\title{
Scaling phenomena driven by inhomogeneous conditions at first-order quantum transitions
}

\author{
Massimo Campostrini, ${ }^{1}$ Jacopo Nespolo, ${ }^{1}$ Andrea Pelissetto, ${ }^{2}$ and Ettore Vicari ${ }^{1}$ \\ ${ }^{1}$ Dipartimento di Fisica dell'Università di Pisa and INFN, Largo Pontecorvo 3, I-56127 Pisa, Italy \\ ${ }^{2}$ Dipartimento di Fisica dell'Università di Roma “La Sapienza” and INFN, Sezione di Roma I, I-00185 Roma, Italy
}

(Received 24 November 2014; published 9 February 2015)

\begin{abstract}
We investigate the effects of smooth inhomogeneities at first-order quantum transitions (FOQTs), such as those arising in the presence of a space-dependent external field, which smooths out the discontinuities of the low-energy properties at the transition. We argue that a universal scaling behavior emerges in the space transition region close to the point in which the external field takes the value for which the homogeneous system undergoes the FOQT. We verify the general theory in two model systems. We consider the quantum Ising chain in the ferromagnetic phase and the $q$-state Potts chain for $q=10$, investigating the scaling behavior which arises in the presence of an additional inhomogeneous parallel and transverse magnetic field, respectively. Numerical results are in full agreement with the general theory.
\end{abstract}

DOI: 10.1103/PhysRevE.91.022108

PACS number(s): 05.30.Rt, 64.60.fd, 64.60.De

\section{INTRODUCTION}

The theories of classical and quantum phase transitions [1-3] generally apply to homogeneous systems. However, homogeneity is often an ideal limit of experimental conditions. Inhomogeneous conditions generally smooth out the singularities at phase transitions. This is also expected at first-order transitions, which are characterized by discontinuities in the thermodynamic quantities in the classical case or in the ground-state properties in the quantum case.

In the presence of smooth inhomogeneities, we may simultaneously observe different phases in different space regions, separated by crossover regions in which the system goes from one phase to another. For example, this behavior is observed in typical cold-atom experiments [4], in which the atoms are constrained in a limited space region by an inhomogeneous (usually harmonic) trap, which effectively makes the chemical potential space dependent.

The effects of the inhomogeneous conditions have been much investigated at continuous transitions [4-46]. For sufficiently smooth inhomogeneities, classical or quantum systems, at classical (finite-temperature) or quantum (zero-temperature) transitions, show a universal scaling behavior in a space region whose size is controlled by the typical length scale $\ell$ of the inhomogeneity. The scaling behavior is universal and somewhat analogous to the standard finite-size scaling (FSS) occurring in homogeneous systems [47-50]. In particular, it only depends on the universality class of the transition occurring in the homogeneous system. There is, however, a crucial difference between the usual FSS and that observed in the presence of an inhomogeneity. In the latter case, there are indeed two relevant length scales. Besides the correlation length $\xi$ there is the length scale $\ell$, related to $\xi$ by a nontrivial power law at the critical point, $\xi \sim \ell^{\theta}$, where $\theta$ is a universal exponent depending on the homogeneous universality class and on some general features of the external space-dependent field $[19,25]$.

Scaling phenomena also emerge at first-order classical transitions in the presence of a temperature gradient [46] or a space-dependent external field. They are observed in the transition region in which the space-dependent temperature assumes values close to the critical temperature of the homogeneous system. Thermodynamic quantities show a universal scaling behavior, characterized by nontrivial power laws, which is quite similar to that observed at continuous transitions.

In this paper we study the effects of inhomogeneous conditions at first-order quantum transitions (FOQTs). FOQTs are of great interest, as they occur in a large number of quantum many-body systems, such as quantum Hall samples [51], itinerant ferromagnets [52], heavy fermion metals [53-55], and so on. They also occur in multicomponent cold-atom systems in optical lattices, with spin-orbit coupling and synthetic gauge fields. These systems show several phases, some of them separated by FOQTs [56-62].

We investigate the scaling behavior arising when one of the model parameters smoothly depends on space. We put forward a scaling theory, which describes the low-energy properties in the crossover space region where the system changes phase. We verify this scaling theory in two relatively simple quantum many-body systems, the quantum Ising and Potts chains in the presence of space-dependent magnetic fields. Numerical results are in full agreement with the theoretical predictions.

The paper is organized as follows. In Sec. II we define the quantum Ising and Potts chains with a space-dependent magnetic field $h_{x}$ and present numerical results for their behavior around the spatial point where $h_{x}=0$, the value at which the FOQT occurs in the homogeneous case. In Sec. III we present a general theory for the scaling behavior in the crossover region close to the transition spatial point. In Sec. IV we check the scaling theory by analyzing numerical results for the Ising and the Potts chain with $q=10$. Finally, in Sec. V we draw our conclusions.

\section{QUANTUM ISING AND POTTS CHAINS}

In order to make the discussion concrete, we first define the quantum models that we use as theoretical laboratories to study FOQTs in the presence of a spatial inhomogeneity. We consider the Ising chain in the ordered phase in the presence of a parallel magnetic field coupled to the order-parameter spin operator and the quantum $q$-state Potts chain with $q=10$ in the presence of a transverse magnetic field. In the homogeneous case, both models undergo a FOQT. For each 
model, we compute several quantities using the density matrix renormalization-group (DMRG) method [63]. Some details of the DMRG implementation can be found in Refs. [64,65], where we presented numerical studies of the same models in homogeneous conditions. From the numerical point of view, simulations of the inhomogeneous system do not present any additional difficulty.

\section{A. The quantum Ising chain}

We consider a quantum Ising chain of size $2 L+1$ with a space-dependent parallel magnetic field $h_{x}$ coupled with the order-parameter spin operator. Its Hamiltonian is

$$
\begin{aligned}
H_{I}= & -J \sum_{x=-L}^{L-1} \sigma_{x}^{(1)} \sigma_{x+1}^{(1)} \\
& -g \sum_{x=-L}^{L} \sigma_{x}^{(3)}-\sum_{x=-L}^{L} h_{x} \sigma_{x}^{(1)},
\end{aligned}
$$

where $\sigma_{x}^{(a)}$ are the Pauli matrices, $g \geqslant 0$ is the transverse magnetic field, $J$ is a coupling that will be taken equal to 1 in the following, and $h_{x}$ is a space-dependent magnetic field, which we write as

$$
h_{x} \equiv h(x / \ell) \text {, }
$$

where $\ell$ is a length scale. The most interesting case corresponds to the linear function

$$
h(x)=x .
$$

Indeed, as we shall discuss, models with $h(x)$ given by Eq. (3) and models with generic functions $h(x)$ such that $h\left(x_{0}\right)=0$ and $h^{\prime}\left(x_{0}\right) \neq 0$ show the same scaling behavior around the point where $h_{x}$ vanishes, provided that we rescale $\ell$ so $h_{x} \approx$ $\left(x-x_{0}\right) / \ell$ for $x \rightarrow x_{0}$. In other words, if $h(x)$ admits the expansion

$$
h(x) \approx a_{1}\left(x-x_{0}\right)+a_{2}\left(x-x_{0}\right)^{2}+\ldots
$$

around the point $x_{0}$, where $h\left(x_{0}\right)=0$, with $a_{1} \neq 0$, then the scaling behavior is identical to that obtained by simply considering the model with $h(x)=a_{1}\left(x-x_{0}\right)$.

It is also convenient to extend the analysis to a more general class of functions,

$$
h(x)=\operatorname{sgn}(x)|x|^{p},
$$

parametrized by $p$. This allows us to crosscheck the scaling theory we shall put forward to describe these phenomena. We study the system in the $L \rightarrow \infty$ limit and investigate the scaling behavior with respect to the length scale $\ell$. In the limit $p \rightarrow \infty$ the only allowed states $|s\rangle$ are those such that $\sigma_{x}^{(1)}|s\rangle=+1$ for $x>\ell$ and $\sigma_{x}^{(1)}|s\rangle=-1$ for $x<-\ell$. Thus, in this limit we obtain the Hamiltonian of an Ising chain of size $2\lfloor\ell\rfloor+1$, with fixed opposite boundary conditions (FOBC). Integrating out all states with $|x|>\ell$ we obtain the
Hamiltonian with a boundary term [64],

$$
\begin{aligned}
H_{I, \mathrm{FOBC}}= & -\sum_{x=-\lfloor\ell\rfloor}^{\lfloor\ell\rfloor-1} \sigma_{x}^{(1)} \sigma_{x+1}^{(1)} \\
& -g \sum_{x=-\lfloor\ell\rfloor}^{\lfloor\ell\rfloor} \sigma_{x}^{(3)}+\left(\sigma_{-\lfloor\ell\rfloor}^{(1)}-\sigma_{\lfloor\ell\rfloor}^{(1)}\right) .
\end{aligned}
$$

The homogeneous Ising chain, i.e., model (1) with a uniform magnetic field $h_{x}=h$, has a continuous transition at $g=$ $1, h=0$, belonging to the two-dimensional Ising universality class. This quantum critical point separates a paramagnetic $(g>1)$ and a ferromagnetic $(g<1)$ phase. In the ferromagnetic phase $g<1$, the parallel magnetic field $h$ drives a FOQT at $h=0$, with a discontinuity of the magnetization, i.e., of the ground-state expectation value of $\sigma_{x}^{(1)}$. Indeed, we have [66]

$$
\begin{gathered}
m_{ \pm}=\lim _{h \rightarrow 0^{ \pm}} \lim _{L \rightarrow \infty}\left\langle\sigma_{x}^{(1)}\right\rangle= \pm m_{c}, \\
m_{c}=\left(1-g^{2}\right)^{1 / 8} .
\end{gathered}
$$

Therefore, in the presence of an inhomogeneous field which vanishes changing sign at $x=0$, such as that defined in Eq. (3), the point $x=0$ effectively corresponds to the spatial point which separates the two oppositely magnetized phases, with $\left\langle\uparrow\left|\sigma_{x}^{(1)}\right| \uparrow\right\rangle=m_{+}$and $\left\langle\downarrow\left|\sigma_{x}^{(1)}\right| \downarrow\right\rangle=m_{-}$.

Numerical DMRG estimates of the local magnetization

$$
m(x)=\left\langle\sigma_{x}^{(1)}\right\rangle
$$

are shown in Figs. 1, 2, and 3 for linearly and quadratically varying fields $h(x)$. The system size $L$ is always large enough to guarantee that the data are in the infinite-size limit. This is easily checked by comparing data with the same $\ell$ and increasing values of $L$. Examples are reported in Figs. 1 and 3. Note that, with increasing $\ell$, smaller and smaller ratios $L / \ell$ are needed to observe the large- $L$ limit around $x=0$. This

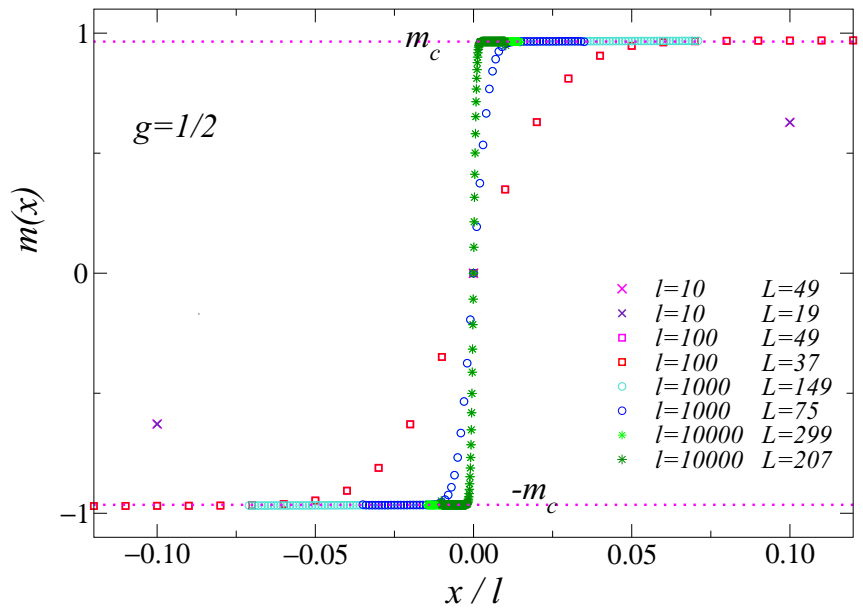

FIG. 1. (Color online) The local magnetization $m(x) \equiv\left\langle\sigma_{x}^{(1)}\right\rangle$ for the Ising chain (1) with $g=1 / 2$ and $h_{x}=x / \ell$ versus $x / \ell$. The dotted lines correspond to the transition values $m_{ \pm}= \pm m_{c}$ given in Eq. (8). The data for the same $\ell$ and different $L$ cannot be distinguished on the scale of the figure, showing that they effectively provide the $L \rightarrow \infty$ limit. 

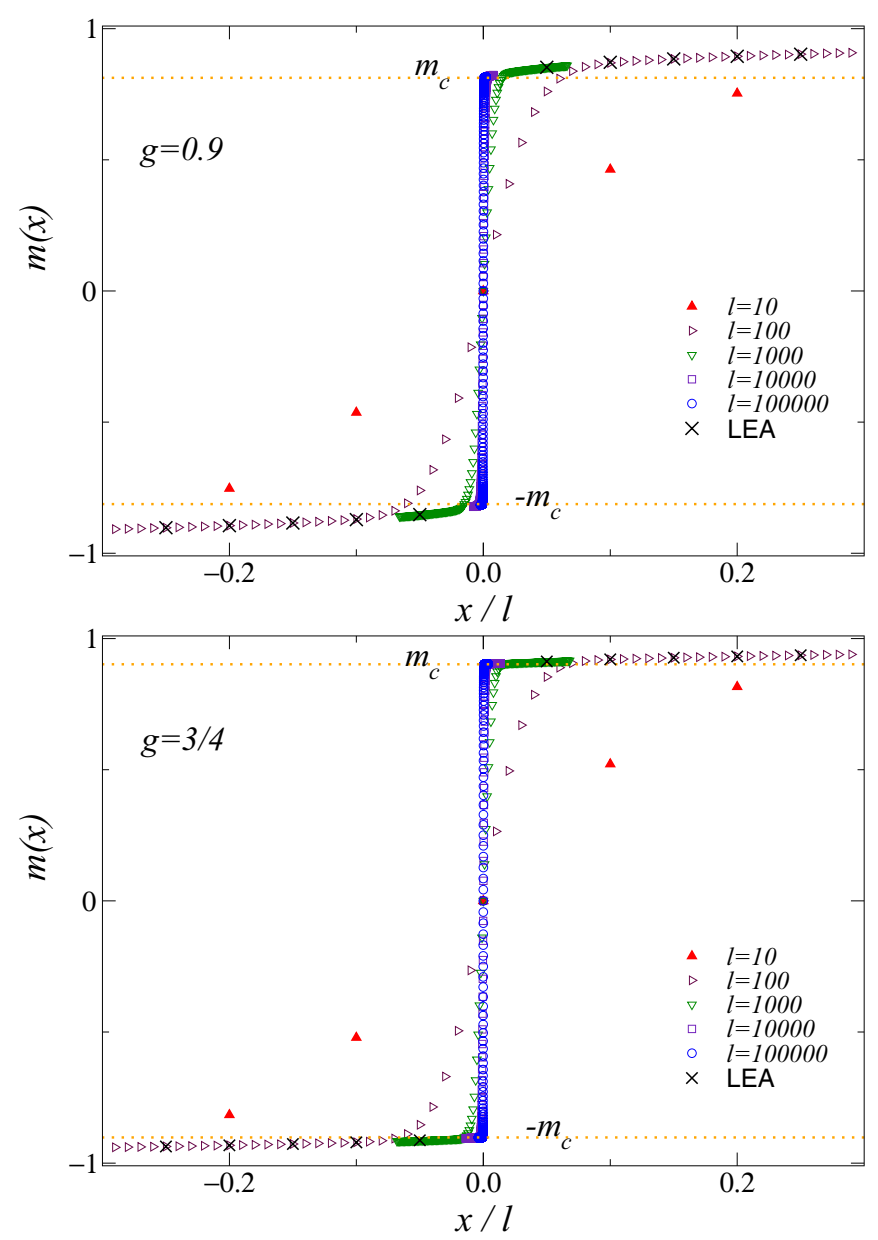

FIG. 2. (Color online) The local magnetization $m(x)$ for model (1) with $h_{x}=x / \ell$ for $g=3 / 4$ (bottom) and $g=9 / 10$ (top). The values of $m_{c}$ are given in Eq. (8). We also show the LEA predictions, obtained by using the DMRG estimates of the magnetization $m_{h}(h)$ as a function of a uniform parallel magnetic field $h$.

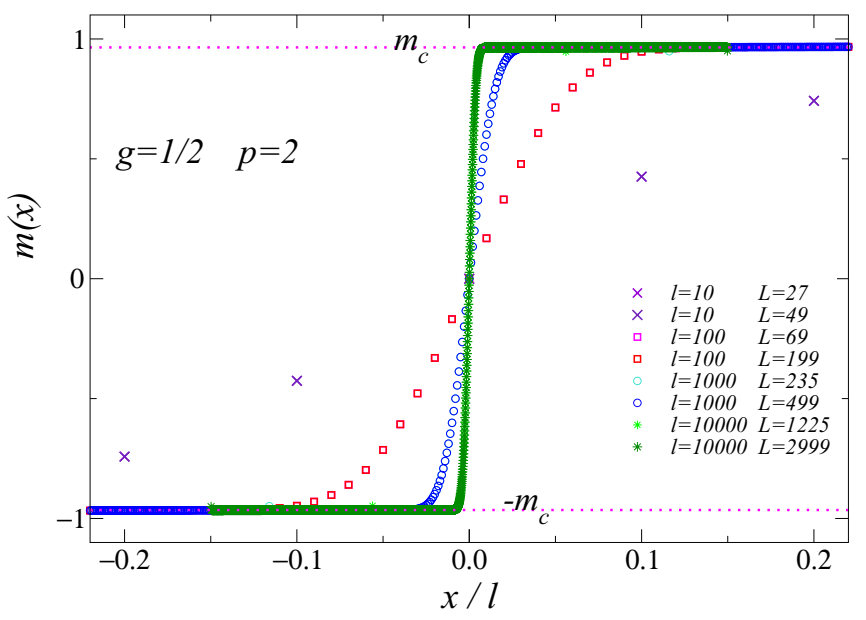

FIG. 3. (Color online) The local magnetization $m(x)$ for model (1) in the presence of a quadratic space-dependent $(p=2) h_{x}$ at $g=1 / 2$. The data for the same $\ell$ and different $L$ practically coincide, hence they provide a good approximation of $m(x)$ in the $L \rightarrow \infty$ limit. is explained by the scaling theory of Sec. III, which shows that the relevant scaling length in the crossover region around $x=0$ is $\xi \sim \ell^{\theta}$ with $\theta=1 / 4$ for $h(x)=x$ and $\theta=2 / 5$ for the $h(x) \sim|x|^{2}$. Therefore, the relevant length ratio in the crossover region is $L / \ell^{\theta}$ and not $L / \ell$, so the infinite- $L$ limit is observed for $L / \ell \gg \ell^{\theta-1} \sim \ell^{-3 / 4}$ for $p=1$ and $L / \ell \gg \ell^{-3 / 5}$ for $p=2$. Therefore, the ratio $L / \ell$ decreases for $\ell \rightarrow \infty$.

The data around $x=0$ show a crossover between the two magnetization values $m_{ \pm}$defined in Eq. (7), which becomes sharper and sharper with increasing $\ell$. The results for $p=1$ and $p=2$, shown in Figs. 1 and 3 for the same value $g=1 / 2$, show a similar behavior. The only difference is the size of the crossover region. As expected, for the same value of $\ell$, it is larger for $p=2$ than for $p=1$.

In noncritical regimes, away from phase transitions, inhomogeneity effects can be effectively taken into account by using the local-equilibrium approximation (LEA). It assumes that the behavior at a given point is only determined by the local values of the Hamiltonian parameters. As a consequence, the behavior in the inhomogeneous system at a given point $x$ is approximately equal to that observed in the homogeneous system for the same values of the Hamiltonian parameters. An example is the local-density approximation, which is widely used to study particle systems with an effective space-dependent chemical potential, see, e.g., Refs. [4,32,67-70]. The LEA assumes the absence of significant space correlations, hence it does not provide a satisfactory description of the system in the presence of classical or quantum transitions, characterized by large-scale correlations [32,39,70]. The failure of the LEA is also observed at first-order classical transition in the presence of a temperature gradient [46].

In our model, the LEA predicts

$$
m(x) \approx m_{\text {lea }}(x / \ell)=m_{h}[h(x / \ell)],
$$

where $m_{h}(h)$ is the infinite-size magnetization in the homogeneous system in the presence of a uniform magnetic field $h$. Note that since the external field $h_{x}$ is a function of the ratio $x / \ell$, the LEA estimate scales as $x / \ell$. The LEA is expected to provide a good approximation when $h_{x}$ varies smoothly, thus for large $\ell$, and far from the transition. In particular, since the magnetization in the homogeneous system takes values in $m_{c} \leqslant|m| \leqslant 1$, the LEA can not be applied in the crossover region where $|m|<m_{c}$.

LEA results are shown in Fig. 2. The numerical data at fixed $x / \ell$ approach the LEA estimate with increasing $\ell$. Convergence is fast far from $x=0$, but it becomes significantly slower when approaching $x=0$. As we shall see, this nonuniform behavior as $|x| \rightarrow 0$ reflects a different nontrivial scaling behavior which characterizes the crossover region around $x=0$ in the limit $\ell \rightarrow \infty$. This is a novel regime, somehow probing the mixed quantum phase where $-m_{c}<m(x)<m_{c}$.

\section{B. The quantum Potts chain}

The quantum $q$-state Potts chain is the quantum counterpart of the classical two-dimensional Potts model [71-73] whose Hamiltonian is

$$
H_{c}=-J \sum_{\langle i j\rangle} \delta\left(s_{i}, s_{j}\right),
$$


where the sum is over the nearest-neighbor sites of a square lattice, $s_{i}$ are spin variables taking $q$ integer values, i.e., $s_{i}=$ $1, \ldots, q$, and $\delta(m, n)=1$ if $m=n$ and zero otherwise. The quantum Hamiltonian can be derived from the time continuum limit of the transfer matrix, with $q$ states per site, which can be labeled by an integer number $|n=1\rangle, \ldots,|n=q\rangle$. For a chain of size $2 L+1$ it reads $[65,74,75]$

$$
H_{P}=-\sum_{x=-L}^{L-1} \sum_{k=1}^{q-1} \Omega_{x}^{k} \Omega_{x+1}^{q-k}-g \sum_{x=-L}^{L} \sum_{k=1}^{q-1} M_{x}^{k}-\sum_{k=1}^{q-1} \Omega_{-L}^{k}
$$

where $\Omega_{x}$ and $M_{x}$ are $q \times q$ matrices:

$$
\begin{gathered}
\Omega_{m n}=\delta_{m n} \omega^{n-1}, \quad \omega=e^{i 2 \pi / q}, \\
M_{m n}=\delta_{\bmod (m, q), n-1}=\left(\begin{array}{cccc}
0 & 1 & & \\
& \ddots & \ddots & \\
& & & 1 \\
1 & & & 0
\end{array}\right) .
\end{gathered}
$$

These matrices commute on different sites and satisfy the algebra: $\Omega_{x}^{k} \Omega_{x}^{l}=\Omega_{x}^{k+l}, M_{x}^{k} M_{x}^{l}=M_{x}^{k+l}, \Omega_{x}^{q}=M_{x}^{q}=\mathbb{I}, M_{x}^{k} \Omega_{x}^{l}=$ $\omega^{k l} \Omega_{x}^{l} M_{x}^{k}$.

The last term in the right-hand side of Eq. (12) is a boundary term which breaks the $q$-state symmetry, favoring the state $n=1$. It ensures the self-dual property [75]

$$
H_{P}(g)=g H_{P}(1 / g)
$$

even for finite chains [65]. The Hamiltonian $H_{P}$ corresponds to a chain with mixed self-dual boundary conditions (SDBC), with a fixed state $n=1$ at site $x=-L-1$ and an unmagnetized disordered state $\propto \sum_{n=1}^{q}|n\rangle$ at $x=L+1$. For $q=2$ Hamiltonian $H_{P}$ corresponds to that of a quantum Ising chain with mixed fixed-free boundary conditions.

Like the quantum Ising chain, the quantum Potts chain shows two phases: a disordered phase for sufficiently large values of $g$ and an ordered phase for small $g$, in which the system magnetizes along one of the $q$ directions. The transition point is easily inferred from the duality relation (15), obtaining $g=g_{c}=1$. For $q>4$ the two phases are separated by a FOQT, where the energy density and the magnetization are discontinuous [72-75]. We define the energy density as

$$
e\left(x_{b}\right)=\left\langle\mathcal{E}_{x}\right\rangle, \quad \mathcal{E}_{x}=\delta\left(n_{x}, n_{x+1}\right)=\frac{1}{q} \sum_{k=1}^{q} \Omega_{x}^{k} \Omega_{x+1}^{q-k},
$$

where $x_{b}=x+1 / 2$ is the position of the bond center. The infinite-volume energy density changes discontinuously across the FOQT, i.e., the two limits

$$
e_{ \pm}=\lim _{g \rightarrow 1^{ \pm}} \lim _{L \rightarrow \infty} e(x)
$$

differ for $q>4$. Their difference $\Delta e \equiv e_{+}-e_{-}$is the analog of the latent heat in first-order finite-temperature transitions. For example [65], for the $q=10$ Potts chain we have $e_{-}=$ $0.8060(1)$ and $e_{+}=0.3745(5)$.

Also the magnetization is discontinuous at the transition, varying from zero in the disorder $(g>1)$ phase to a nonzero value in the ordered $(g<1)$ phase. We define the local magnetization of the ground state as

$$
\begin{gathered}
m(L, g, x)=\left\langle\mathcal{M}_{x}\right\rangle, \\
\mathcal{M}_{x}=\frac{q \delta\left(n_{x}, 1\right)-1}{q-1}, \quad \delta\left(n_{x}, 1\right)=\frac{1}{q} \sum_{k=1}^{q} \Omega_{x}^{k} .
\end{gathered}
$$

The limit

$$
m_{c}=\lim _{g \rightarrow 1^{-}} \lim _{b \rightarrow 0} \lim _{L \rightarrow \infty} m(x)
$$

is nonzero for $q>4$, where $b$ is a magnetic field coupled to the projector onto the $n=1$ state, i.e., associated with the Hamiltonian term

$$
H_{P b}=-b \sum_{x=-L}^{L} \sum_{k=1}^{q} \Omega_{x}^{k} .
$$

$m_{c}$ is known exactly [76,77]: $m_{c}=0.857107 \ldots$ for $q=10$ [78].

We generalize the homogeneous model (12), considering a space-dependent transverse magnetic field. We fix $g=g_{c}=1$ and add to Hamiltonian (12) the term

$$
H_{P h}=-\sum_{x=-L}^{L} h_{x} \sum_{k=1}^{q-1} M_{x}^{k}
$$

obtaining an inhomogeneous system with a transverse magnetic field $1+h_{x}$ at point $x$. With this choice, the transverse field is 1 at the center $x=0$ of the chain, which is therefore the point where the phase change occurs. Moreover, we consider $L \leqslant \ell$ so the local transverse field satisfies $1+h_{x}>0$ for all values of $x$. Again we consider different functions $h(x)$, as in Eq. (3) or Eq. (5). For $p \rightarrow \infty$, we recover the homogeneous system with SDBC.

In Fig. 4 we show DMRG estimates of the local magnetization $m(x)$ for $q=10$ in the presence of a magnetic

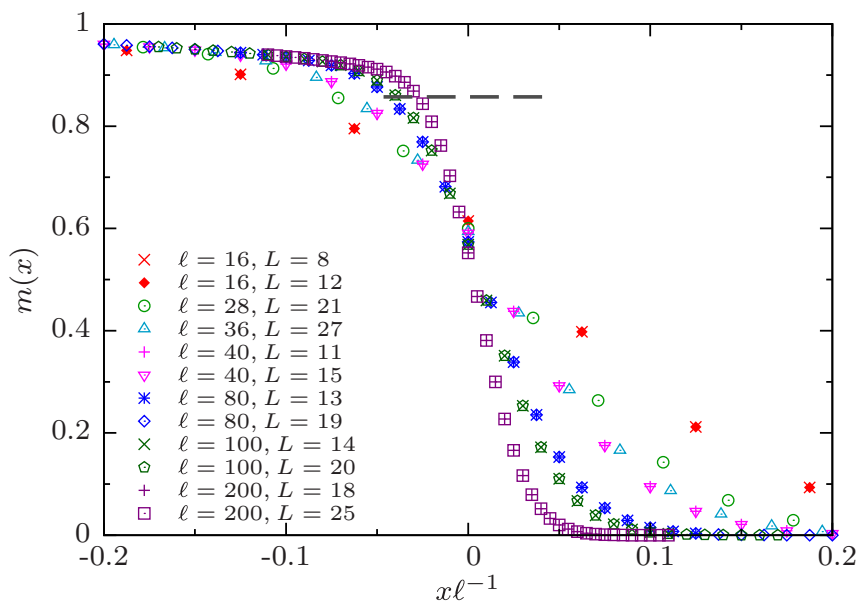

FIG. 4. (Color online) The local magnetization $m(x)$ for the quantum $q=10$ Potts chain in the presence of a linearly varying transverse field. The dashed line correspond to the value $m_{c} \approx 0.8571$ defined in Eq. (20). The data for the same $\ell$ and different $L$ practically coincide, showing that they are already asymptotic and do not depend on $L$. 


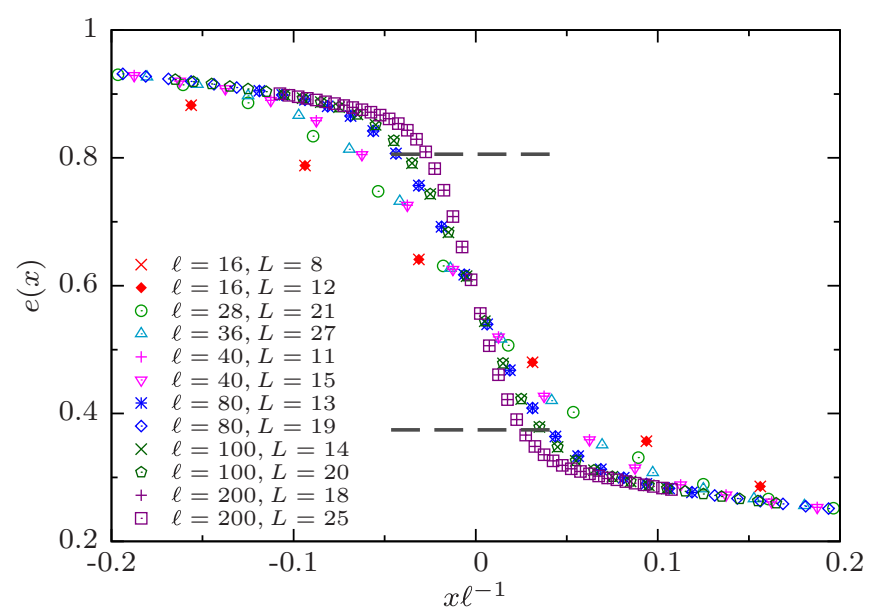

FIG. 5. (Color online) The energy density $e(x)$ defined in Eq. (16) for the quantum $q=10$ Potts chain in the presence of a linearly varying transverse field. The dashed lines correspond to $e_{-}=0.8060$ and $e_{+}=0.3745$ defined in Eq. (17). The data for the same $\ell$ and different $L$ practically coincide, showing that they are already asymptotic and do not depend on $L$.

field linearly varying with $x$ as in Eq. (3). As expected, the magnetization rapidly drops in the space region corresponding to the disordered phase, i.e., $x>0$. Data for the local energy density are shown in Fig. 5. They clearly show a crossover region in which $e(x)$ varies rapidly from $e_{-}$to $e_{+}$, which are the values of the energy density in the ordered and disordered phases, respectively.

Like the Ising case, the estimates of the different observables close to $x=0$, i.e., for sufficiently small ratios $x / \ell$, rapidly converge when increasing the ratio $L / \ell$ keeping $\ell$ fixed. Estimates of $m(x)$ and $e(x)$ are shown in Figs. 4 and 5. As for the energy differences between the lowest states, for $\ell=200$ we have $E_{1}-E_{0}=0.90956$ for $L=18$ and $E_{1}-E_{0}=0.90952$ for $L=25$. As before, with increasing $\ell$, smaller and smaller ratios $L / \ell$ are sufficient to effectively obtain $L$-independent results. This is essentially related to the fact that the relevant scaling length in the crossover region is $\xi \sim \ell^{\theta}$ with $\theta=1 / 3$ for linear $h(x)$, as we will discuss in Sec. III.

Note that since the Potts chain with $q=10$ is much more complex than the Ising chain, DMRG computations allow us to get reliable results only for smaller chain sizes and therefore not-too-large length scales of the external magnetic field. This is essentially related to the fact that many more states per site must be kept in the computations.

\section{SCALING BEHAVIOR IN THE CROSSOVER SPACE REGION}

In this section we present a scaling theory for the behavior observed at FOQTs in the presence of inhomogeneous external fields, whose $x$ dependence is given in Eqs. (2) and (5) for generic values of $p$.
Before discussing the general theory, we consider the case $p \rightarrow \infty$. In this limit we obtain

$$
\begin{array}{ll}
h_{x}=+\infty & \text { for } x>\ell \\
h_{x}=0 & \text { for }-\ell<x<\ell \\
h_{x}=-\infty & \text { for } x<-\ell .
\end{array}
$$

Therefore, in this limit the infinite-size inhomogeneous system is equivalent to a finite-size homogeneous system defined for $-\lfloor\ell\rfloor \leqslant x \leqslant\lfloor\ell\rfloor$, with appropriate boundary conditions that depend on the quantity coupled to the field $h_{x}$. In the Ising case one obtains FOBC, i.e., Hamiltonian (6), while in the Potts case one obtains SDBC, i.e., Hamiltonian (12) with $h_{x}=0$. Therefore, in the $p \rightarrow \infty$ limit the scaling behavior predicted by the general theory for generic values of $p$ must reproduce the finite-size behavior of the corresponding homogeneous system. Although at FOQTs there is no diverging correlation length in the infinite-volume limit, one can observe FSS close to the transition point, both in the case of classical and quantum first-order transitions [64,65,75,79-90].

The relevant FSS variable at a FOQT is the ratio $\kappa=$ $E_{L} / \Delta_{L}$ between the energy contribution $E_{L}$ of the perturbation driving the transition and the energy difference (gap) of the lowest states $\Delta_{L} \equiv E_{1}-E_{0}$ at the transition point. The $L$ dependence of the gap $\Delta_{L}$ depends on the model and on the specific boundary conditions considered-a very specific feature of FOQTs. In general, we parametrize this dependence as

$$
\Delta_{L} \sim L^{-z}
$$

introducing a dynamic exponent $z$. Assuming that the perturbation is uniform, the energy associated with the perturbation scales as $E_{L} \sim h L^{d}$, where $d$ is the space dimension. It follows that

$$
\kappa \sim h L^{d+z}
$$

In the language of renormalization-group (RG) theory, this relation allows us to associate a $\mathrm{RG}$ dimension with the perturbation $h$, given by

$$
y_{h}=d+z .
$$

The above-reported considerations can be generalized to space-dependent quantities. The scaling variable is $x / L$ or, equivalently,

$$
x h^{1 / y_{h}}
$$

given that $L \sim h^{-1 / y_{h}}$ in the FSS limit.

We wish now to extend these FSS scaling relations [64] to the inhomogeneous case. In the presence of inhomogeneous fields whose space behavior is given by Eq. (5), FSS can be heuristically derived by replacing the parameter $h$ with $h_{x} \sim$ $(x / \ell)^{p}$ in Eq. (27). Therefore, assuming that $L$ is sufficiently large not to play any role, we obtain the scaling variable

$$
x\left(\frac{x}{\ell}\right)^{p / y_{h}}=\left(\frac{x}{\ell^{\theta}}\right)^{1+p / y_{h}},
$$

where the exponent $\theta$ is given by

$$
\theta=\frac{p}{p+y_{h}} .
$$


Therefore, in the presence of an inhomogeneous external field FSS is obtained by taking $x \rightarrow 0, \ell \rightarrow \infty$, keeping

$$
X=x / \ell^{\theta}
$$

fixed. Relation (30) expresses the fact that, in the crossover region around the transition point, a new length scale $\xi$, behaving as

$$
\xi \sim \ell^{\theta},
$$

controls the crossover. Note that $\theta \rightarrow 1$ for $p \rightarrow \infty$, consistently with the fact that one must recover the FSS of the homogeneous system in this limit. It is important to stress that definition (30) assumes that $h(x=0)$ is the value of the field for which the transition occurs. If criticality occurs for $h=h_{x_{0}}$, then the scaling variable is $X=\left(x-x_{0}\right) \ell^{-\theta}$.

For the Ising chain with FOBC and for $g<1$ (ferromagnetic phase) the gap behaves as [64] $L^{-2}$, so

$$
z=2, \quad y_{h}=3, \quad \theta=\frac{p}{p+3} .
$$

In particular, we have $\theta=1 / 4,2 / 5$ for $p=1,2$, respectively. In the case of the Potts chain with SDBC one finds instead [65] $\Delta_{L} \sim L^{-1}$, so

$$
z=1, \quad y_{h}=2, \quad \theta=\frac{p}{p+2} .
$$

In particular, we have $\theta=1 / 3,1 / 2$ for $p=1,2$, respectively.

The previous results allow us to predict the scaling behavior of the different quantities for $\ell \rightarrow \infty$. The energy difference $\Delta_{\ell}$ of the two lowest levels is expected to scale as

$$
\Delta_{\ell} \sim \xi^{-z} \sim \ell^{-z \theta},
$$

where $\theta$ is given in Eq. (29). Analogously, the local magnetization is expected to asymptotically behave as

$$
m(x)=m_{c} f_{m}(X), \quad X=x / \ell^{\theta},
$$

where $m_{c}$ is a nonuniversal normalization constant, which can be identified with the value of the magnetization in one of the magnetized phases. In the Ising and Potts case we identify $m_{c}$ with the quantities reported in Eqs. (7) and (20), so $\lim _{X \rightarrow-\infty} f_{m}(X)=-1$ in the Ising case and $\lim _{X \rightarrow-\infty} f_{m}(X)=1$ in the Potts case. We also consider the two-point function of the order parameter. In the Ising case it is defined as

$$
G(x, y)=\left\langle\sigma_{x}^{(1)} \sigma_{y}^{(1)}\right\rangle,
$$

while in the Potts case we consider the function

$$
G(x, y)=\left\langle\mathcal{M}_{x} \mathcal{M}_{y}\right\rangle,
$$

and its connected part,

$$
G_{c}(x, y)=\left\langle\mathcal{M}_{x} \mathcal{M}_{y}\right\rangle-\left\langle\mathcal{M}_{x}\right\rangle\left\langle\mathcal{M}_{y}\right\rangle
$$

where $\mathcal{M}_{x}$ is defined in Eq. (19). For $\ell \rightarrow \infty$, in the crossover region $x \sim \ell^{-\theta}$, we expect the scaling behavior

$$
G\left(x_{1}, x_{2}\right) \approx m_{c}^{2} f_{g}\left(X_{1}, X_{2}\right) .
$$

An analogous scaling is expected for its connected part $G_{c}\left(x_{1}, x_{2}\right)$. The scaling functions $f_{m}$ and $f_{g}$ are expected to be universal, i.e., independent of the microscopic details of the model. For example, in the case of the Ising chain, they are expected to be independent of $g$ (as long as $g<1$, so the system is in the ordered phase), apart from a rescaling of the arguments.

When the energy density is discontinuous at the FOQT, as in the Potts case, we expect that the local energy density scales as

$$
e(x) \approx f_{e}(X) .
$$

The scaling function $f_{e}$ is expected to satisfy

$$
\lim _{X \rightarrow \pm \infty} f_{e}(X)=e_{ \pm}
$$

where $e_{ \pm}$are defined in Eq. (17), because it describes the crossover between the two pure phases in which the energy density of the system takes the values $e_{ \pm}$. We note that analogous scaling behaviors have been conjectured, and numerically checked, at classical first-order transitions in the presence of a temperature gradient [46].

All scaling expressions hold for $\ell \rightarrow \infty$. For finite values of $\ell$, scaling corrections occur. Numerical data are always consistent with corrections decaying as $\ell^{-\theta}$. A posteriori, such a behavior can be interpreted as a boundary effect. Indeed, for a finite-size homogeneous system, scaling corrections for the boundary conditions that we have discussed, i.e., those obtained in the limit $p \rightarrow \infty$, decay as $1 / L$. In the inhomogeneous case, the role of $L$ is played by $\ell^{\theta}$ in all scaling expressions, hence it looks natural to expect $\ell^{-\theta}$ corrections, as confirmed numerically.

It is important to note that $\theta<1$ for all finite values of $p$, so in the crossover limit at fixed $X$, we have $x / \ell=X \ell^{-1+\theta} \rightarrow 0$. This implies that the scaling behavior is completely determined by the small- $x$ behavior of $h(x / \ell)$. For instance, we obtain the same scaling behavior for any $h(x)$ with $h(0)=0$ and $h^{\prime}(0) \neq 0$, as we already discussed in Sec. II A. Higher-order terms give rise to $O\left(\ell^{-1+\theta}\right)$ corrections. In the language of the RG theory, they are irrelevant.

The theory we have presented also provides a general method to determine the value of the external field at which the FOQT occurs. Consider indeed a general model with Hamiltonian

$$
H=H_{0}+g \sum_{x} O_{x}
$$

that has a FOQT for $g=g_{c}$. If $g_{c}$ is not known, it can be determined by studying the inhomogeneous system with the Hamiltonian

$$
H=H_{0}+\sum_{x} g_{x} O_{x},
$$

with $g_{x}=g_{0}+x / \ell$ in the large- $\ell$ limit. Let us describe the method. If $A$ is a quantity that is discontinuous at the transition and $A(x, \ell)$ the corresponding local quantity [in the Potts case, one might consider, for instance, $e(x)]$, the crossover scaling relation is

$$
A(x, \ell)=A_{0} f\left[\left(x-x_{0}\right) / \ell^{\theta}\right]+B \ell^{-\theta} f_{1}\left[\left(x-x_{0}\right) / \ell^{\theta}\right],
$$

neglecting additional corrections. Here $x_{0}$ is such that $g_{x_{0}}=g_{c}$ [hence, $x_{0}=\ell\left(g_{c}-g_{0}\right)$ ], and $A_{0}$ and $B$ are normalization constants. Equation (44) is valid close to $x_{0}$ and for large $\ell$. Since $x_{0}$ corresponds to the transition coupling, $A(x, \ell)$ assumes different values for $x>x_{0}$ and $x<x_{0}$, changing 
rapidly in an interval of size $\ell^{\theta}$ around $x_{0}$. In particular, as evident from Figs. 1-5, the curves corresponding to different values of $\ell$ have an intersection for $x$ close to $x_{0}$. We can exploit this property to determine $g_{c}$ (this is the analog of the Binder cumulant procedure often used in homogeneous critical systems). Indeed, given two different values of $\ell, \ell_{1}$, and $\ell_{2}$, we determine $\bar{x}$ in the crossover region so

$$
A\left(\bar{x}, \ell_{1}\right)=A\left(\bar{x}, \ell_{2}\right) .
$$

If we choose $A_{0}$ and $B$ so $f(y)=1+a y+O\left(y^{2}\right)$ and $f_{1}(y)=$ $1+O(y)$ for small $y$, we obtain

$$
\bar{x}=x_{0}-\frac{B}{A_{0} a}+O\left(\ell^{-\theta}\right) .
$$

It follows that

$$
g_{\bar{x}}=g_{0}+\frac{\bar{x}}{\ell} \approx g_{c}-\frac{B}{A_{0} a \ell} .
$$

In other words, $g_{\bar{x}}$ converges to $g_{c}$ with corrections of order $1 / \ell$, providing an estimate of the position of the transition.

\section{SCALING PHENOMENA INDUCED BY THE INHOMOGENEOUS FIELDS}

In this section we verify the general theory presented in Sec. III, considering the Ising chain and the Potts chain with $q=10$ in the presence of inhomogeneous magnetic fields. We only study the scaling behavior with respect to the length scale $\ell$ in the infinite-size limit. As already discussed in Sec. II, the data we present correspond to values of $L$ large enough to provide accurate estimates of the infinite-size behavior.

\section{A. Results for the Ising chain}

Figure 6 shows the energy difference $\Delta_{\ell}$ of the two lowest states for $p=1,2$ and for the homogeneous system with FOBC $(p \rightarrow \infty)$ as a function of $\ell$. They confirm the behavior (34) with $z=2$, i.e., $\Delta_{\ell} \sim \ell^{-2 \theta}$.

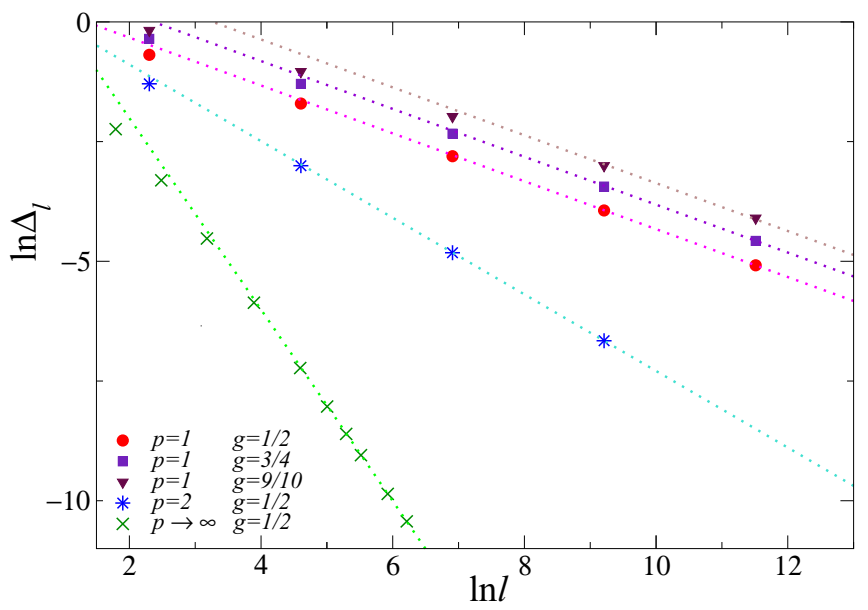

FIG. 6. (Color online) The gap $\Delta_{\ell}$ as a function of $\ell$ for Hamiltonian (1) for inhomogeneous magnetic fields $h_{x}$ with $p=1, p=2$ and for the homogeneous case $(p \rightarrow \infty)$. The dotted lines correspond to the expected behavior $\Delta \sim \ell^{-2 \theta}$ with $\theta=1 / 4$ for $p=1, \theta=2 / 5$ for $p=2$, and $\theta=1$ for $p=\infty$.
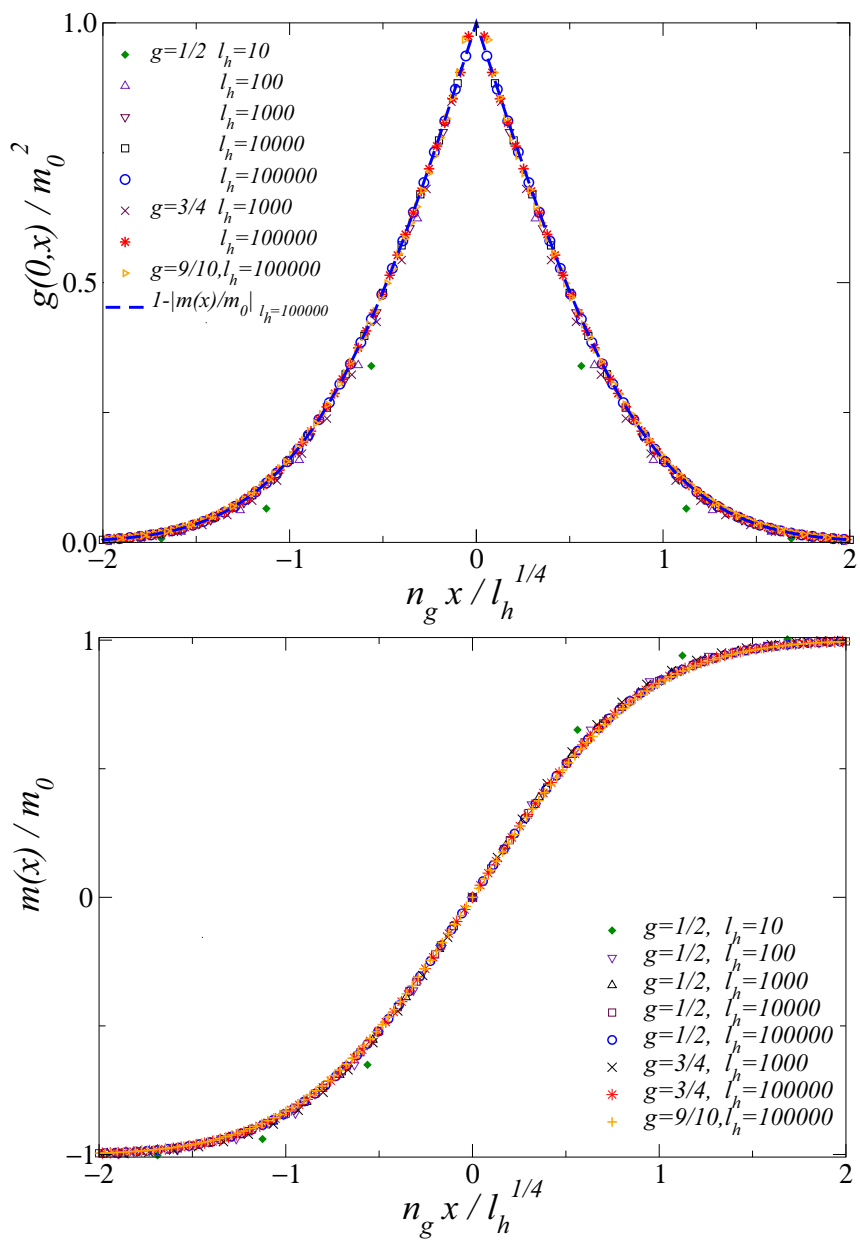

FIG. 7. (Color online) Local magnetization $m(x)$ and two-point function $G(0, x)$ for Hamiltonian (1) at $g=1 / 2,3 / 4,9 / 10$, with a linearly varying magnetic field $h_{x}=x / \ell$. We plot the ratios $m(x) / m_{c}$ (bottom) and $G(0, x) / m_{c}^{2}$ (top) versus $n_{g} x / \ell^{1 / 4}$, where $n_{g}$ is a normalization factor. We use $n_{g} \approx 1,3 / 4,4 / 7$ for $g=$ $1 / 2,3 / 4,9 / 10$, respectively. In the top panel, the data are in full agreement with $1-\left|m(x) / m_{c}\right|$, supporting prediction (53).

In Fig. 7 we show the local magnetization $m(x)$ and the twopoint function $G(0, x)$ for $p=1$-in this case $\theta=1 / 4$-for three values of $g, g=1 / 2,3 / 4,9 / 10$. They nicely confirm the asymptotic scaling relations (35) and (39) and the universality of the scaling functions $f_{m}$ and $f_{g}$ with respect to $g$. All data fall onto a single curve, provided one appropriately rescales $X$ for each value of $g$.

As in the homogeneous system with FOBC, also in the inhomogeneous case we expect the lowest energy states to be associated with kink states $\left|x_{0}\right\rangle$, i.e., with configurations that have a positive local magnetization for $x>x_{0}$ and a negative local magnetization for $x<x_{0}$. In homogeneous systems $[64,91]$ the states $\sum_{x} e^{i p x}|x\rangle$ with momenta $p$ of order $L^{-1}$ are eigenstates of the Hamiltonian with FOBC, with energies that differ by terms of order $1 / L^{2}$ from that of the ground state. We expect these states to provide the lowest eigenstates even in the presence of a linearly varying magnetic field $h_{x}=x / \ell$. In particular, we expect-numerical data are fully consistent with this conjecture, as we discuss below-that 


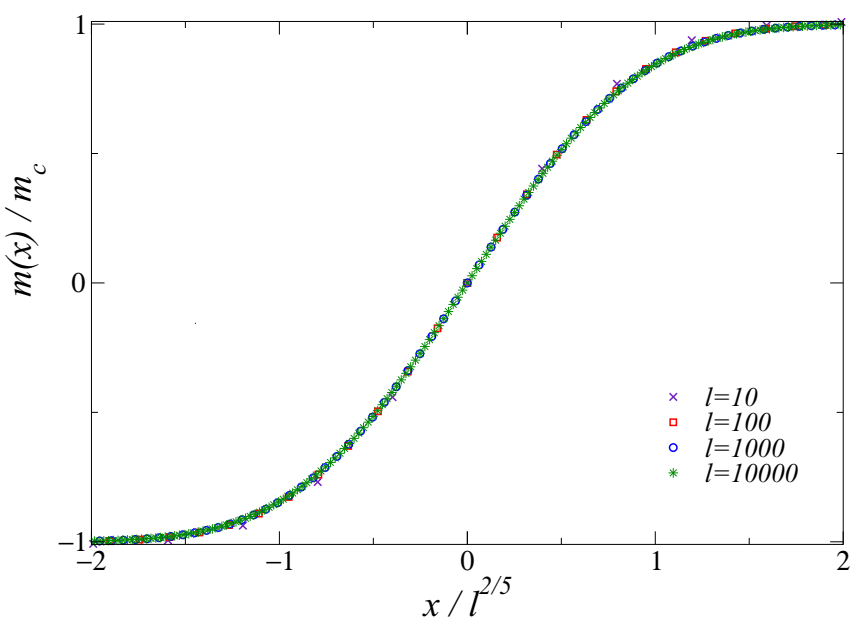

FIG. 8. (Color online) Rescaled local magnetization $m(x) / m_{c}$ versus $x / \ell^{2 / 5}$ for Hamiltonian (1) at $g=1 / 2$ with a quadratically varying magnetic field, see Eq. (5) with $p=2$. As in the linear case $p=1$, the asymptotic relation (53) is satisfied by the data.

the kink states are the relevant ones in the crossover region where Eqs. (35) and (39) are supposed to hold. If kinks control the low-energy behavior, the local magnetization $m(x)$ and the two-point function $G(0, x)$ must be asymptotically related. The argument goes as follows. Given a kink state $\left|x_{0}\right\rangle$, we assume that

$$
\begin{aligned}
& \sigma_{x}^{(1)}\left|x_{0}\right\rangle=-m_{c}\left|x_{0}\right\rangle \quad \text { for } x<x_{0} \\
& \sigma_{x}^{(1)}\left|x_{0}\right\rangle=m_{c}\left|x_{0}\right\rangle \quad \text { for } x>x_{0} .
\end{aligned}
$$

We do not expect these relations to be exact, but we only need them to be valid asymptotically for $\ell \rightarrow \infty$, apart from $x$-dependent corrections of order $\ell^{-\theta}$. Then we assume that the ground state is a superposition of kink states $\left|x_{0}\right\rangle$ and define $p\left(x_{1}, x_{2}\right)$ as the probability of finding a kink state $\left|x_{0}\right\rangle$ with

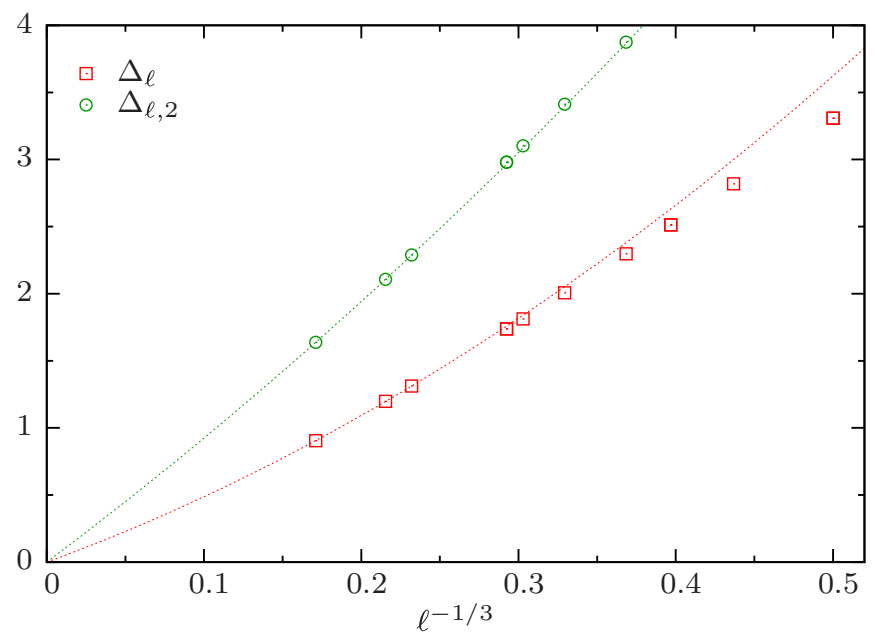

FIG. 9. (Color online) The $\ell$ dependence of the energy differences of the lowest states, i.e., $\Delta_{\ell}=E_{1}-E_{0}$ and $\Delta_{\ell, 2}=E_{2}-E_{0}$. They are consistent with an asymptotic $\ell^{-1 / 3}$ behavior, as predicted by Eq. (34) with $\theta=1 / 3$ and $z=1$. The dotted lines show fits of the data for the largest chains to $c_{1} \ell^{-1 / 3}+c_{2} \ell^{-2 / 3}$.

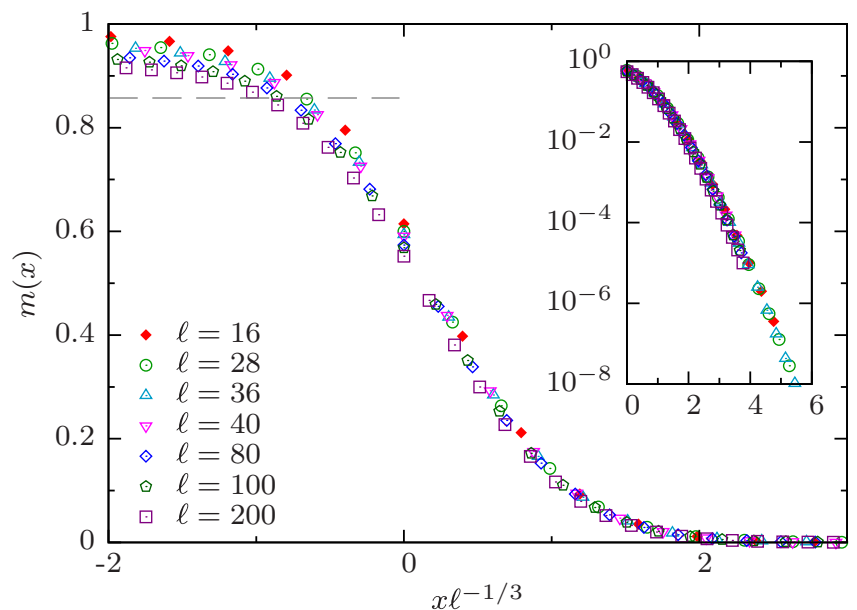

FIG. 10. (Color online) Local magnetization $m(x)$ for the $q=10$ Potts chain in the presence of a linearly varying magnetic field $h_{x}$. The dashed line corresponds to the asymptotic value $m_{c}$. This is slowly approached by the data, due to the expected $O\left(\ell^{-1 / 3}\right)$ corrections. The convergence is much faster for $x>0$. The inset shows the data for $x>0$ in logarithmic scale.

$x_{0}$ belonging to the interval $\left(x_{1}, x_{2}\right)$. Then it is immediate to obtain

$$
m(x)=\left\langle\sigma_{x}^{(1)}\right\rangle=m_{c} p(-\infty, x)-m_{c} p(x, \infty)
$$

so

$$
f_{m}(X)=\frac{m(x)}{m_{c}}=2 p\left(-\infty, X \ell^{\theta}\right)-1
$$

Let us now consider the two-point function $G\left(x_{1}, x_{2}\right)$. If $x_{2}>$ $x_{1}$ we obtain

$$
\begin{aligned}
G\left(x_{1}, x_{2}\right) & =m_{c}^{2}\left[p\left(-\infty, x_{1}\right)-p\left(x_{1}, x_{2}\right)+p\left(x_{2}, \infty\right)\right] \\
& =m_{c}^{2}\left[1-2 p\left(x_{1}, x_{2}\right)\right] \\
& =m_{c}^{2}\left[1-2 p\left(-\infty, x_{2}\right)+2 p\left(-\infty, x_{1}\right)\right] .
\end{aligned}
$$

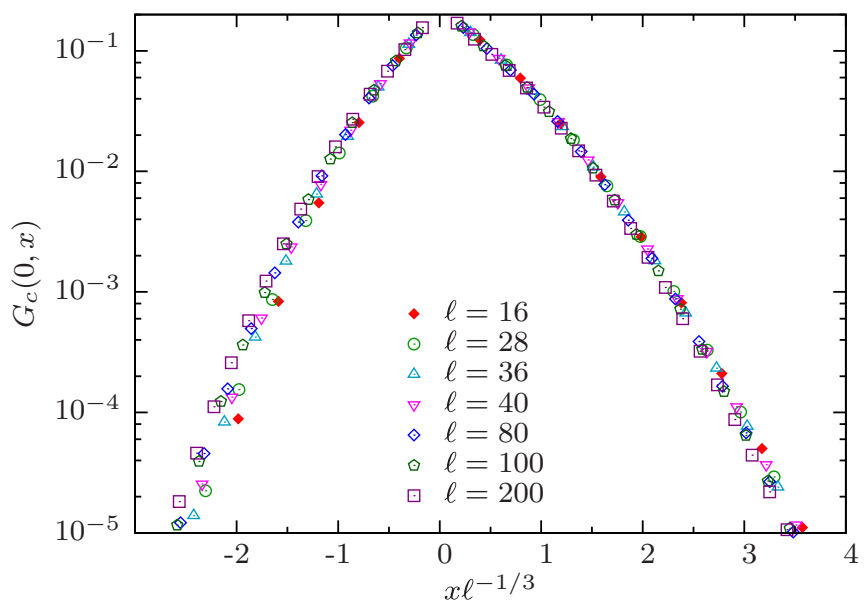

FIG. 11. (Color online) Connected two-point function $G_{c}(0, x)$, defined in Eq. (38), for the $q=10$ Potts chain in the presence of a linearly varying magnetic field $h_{x}$. 


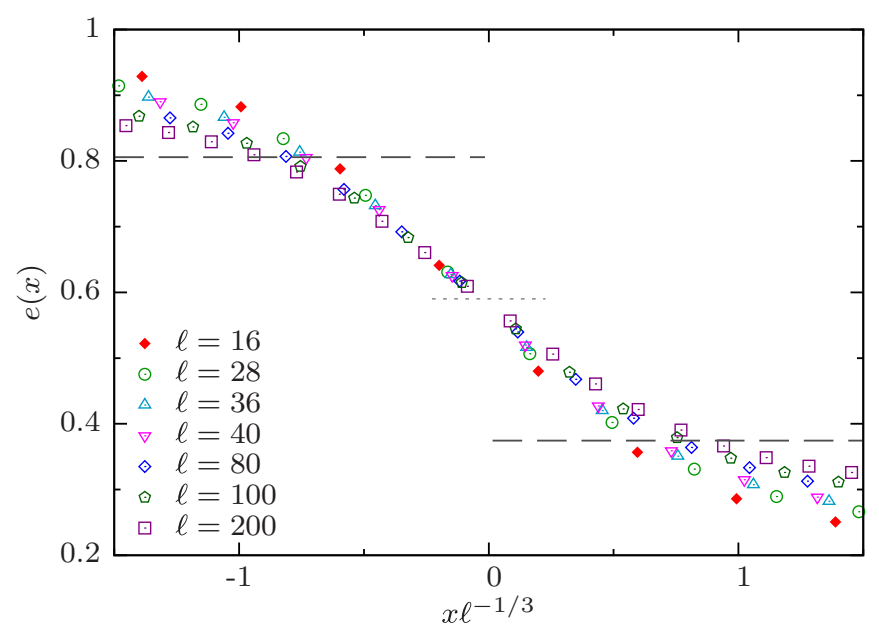

FIG. 12. (Color online) Local energy density for the $q=10$ Potts chain in the presence of a linearly varying magnetic field $h_{x}$. The data appear to approach an asymptotic scaling curve supporting the scaling behavior (40). The dashed lines show the expected asymptotic values $e_{ \pm}$of the scaling function $f_{e}$, cf. Eq. (41). The central dotted line indicates the average value $e_{a}=\left(e_{+}+e_{-}\right) / 2$, which seems to be approached by the data at $x=0$. Scaling corrections are clearly observed, in particular far from the center; they are consistent with the expected $\ell^{-1 / 3}$ behavior.

Using Eq. (50) we obtain finally

$$
f_{g}\left(X_{1}, X_{2}\right)=1-\left|f_{m}\left(X_{2}\right)-f_{m}\left(X_{1}\right)\right|,
$$

where we have added the absolute sign to relax the condition $X_{2}>X_{1}$. For $X_{1}=0$ it becomes

$$
f_{g}(0, X)=1-\left|f_{m}(X)\right| .
$$

Numerical data satisfy this relation, see the top panel of Fig. 7, confirming that kink states control the low-energy behavior of the model.

Analogous results are obtained in the case of quadratic space dependence, i.e., for $p=2$, see Fig. 8. As already mentioned, the scaling behaviors in the $p \rightarrow \infty$ limit must reproduce the FSS of the Ising chain with FOBC [64]. In particular, for any $g<1$ and $h=0$, the FSS function of the local magnetization is given by [92]

$$
f_{m}(X)=X+\frac{1}{\pi} \sin (\pi X), \quad X=x / \ell,
$$

in the large- $\ell$ limit keeping $X$ fixed, with $-1 \leqslant X \leqslant 1$. The two-point function satisfies Eq. (52).

\section{B. Results for the $q=10$ Potts chain}

We now present an analogous analysis of the DMRG data of the $q=10$ Potts chain with a linearly varying field $h_{x}$, cf. Eq. (22). In this case we have that $\theta=1 / 3$ according to Eq. (29).

The energy difference of the lowest states is expected to decrease as $\ell^{-1 / 3}$, as predicted by Eq. (34) with $\theta=1 / 3$ and $z=1$. This is supported by the analysis of the energy differences $\Delta_{\ell}=E_{1}-E_{0}$ and $\Delta_{\ell, 2}=E_{2}-E_{0}$. As shown in
Fig. 9, the data are consistent with an asymptotic behavior

$$
\Delta_{\ell, \#} \approx c_{1} \ell^{-1 / 3}+c_{2} \ell^{-2 / 3}+\ldots
$$

The data reported in Figs. 10 and 11 for the local magnetization $m(x)$ and the two-point function $G(0, x)$, respectively, appear to approach asymptotic curves when they are plotted versus $x / \ell^{\theta}$, supporting the scaling behaviors (35) and (39). Scaling corrections are also clearly observed: They apparently decrease as $\ell^{-\theta}$. Figure 12 shows the energy density, whose behavior is consistent with the scaling ansatz (40), derived in Sec. III, and with Eq. (41).

Let us finally note the similarity of these scaling behaviors with those observed at the first-order classical transition of two-dimensional Potts models in the presence of a temperature gradient along one of the spatial directions, with the other one taken to infinity [46]. Actually, this should not be considered as unexpected, because the quantum Potts chain and the classical two-dimensional Potts model are somehow related by a quantum-to-classical mapping.

\section{CONCLUSIONS}

We have shown that scaling phenomena emerge at FOQTs in the presence of inhomogeneous conditions, such as those arising from a space-dependent external field, e.g., $h_{x} \approx x / \ell$, where $\ell$ is a length scale. They occur in the transition region where the space-dependent parameter $h(x)$ assumes the value $h_{c}$ corresponding to the FOQT of the homogeneous system.

We put forward a general scaling theory to describe the behavior in the crossover space region where the system effectively changes its phase and the discontinuities, that are typical of FOQTs, are smoothed out, i.e., where the system is effectively probing the mixed phase. This scaling behavior is characterized by a critical exponent $\theta$, defined in Eq. (29), which relates the spatial extension $\xi$ of the crossover region with the length scale $\ell$ of the inhomogeneous field, i.e., $\xi \sim \ell^{\theta}$. The exponent $\theta$ depends on some general features of the external field giving rise to the inhomogeneity, such as the effective power law that is satisfied by the space dependence at the transition point and on how it is coupled to the system variables. This scaling behavior is such that the singularities characterizing the FOQT are recovered in the limit $\ell \rightarrow \infty$, where the system becomes homogeneous. We always have $\theta<1$, the value $\theta=1$ being obtained for $p \rightarrow \infty$. In this limit the inhomogeneous scaling behavior matches that of the homogeneous system with appropriate boundary conditions $[64,65]$.

We verify the general theory for two classes of FOQTs. We consider the quantum Ising chain in the ferromagnetic phase, which undergoes a FOQT driven by a parallel magnetic field, and the $q$-state Potts chain with $q=10$, which undergoes a FOQT driven by an even parameter, giving rise to a discontinuity in the ground-state energy density.

Our approach is quite general: The results can be straightforwardly extended to other systems undergoing FOQTs and to other types of inhomogeneities smoothing out the singularities of the transition.

These peculiar inhomogeneous scaling phenomena should be observable in experiments of physical systems, as they only require the measure of local quantities and the control/tuning of the length scale of the inhomogeneity. Such conditions may 
be realized in cold-atom experiments, in particular in optical lattices, when the atomic system is such to have a FOQT in homogeneous conditions, but the space dependence of the effective chemical potential (arising from the trap) smooths out its discontinuities. Around this region we should observe a crossover region with the scaling features put forward in this paper. For example, FOQT lines are expected in the zerotemperature phase diagrams of atomic systems described by multicomponents Bose-Hubbard models [4], with spin-orbit coupling and synthetic gauge fields, see, e.g., Refs. [56-62].
[1] L. D. Landau and E. M. Lifshitz, Statistical Physics (Pergamon Press, Oxford, New York, 1969).

[2] K. G. Wilson, in Nobel Lectures in Physics 1981-1990, edited G. Ekspong (World Scientific, Singapore, 1993); K. G. Wilson and J. Kogut, Phys. Rep. 12, 77 (1974).

[3] S. Sachdev, Quantum Phase Transitions, 2nd ed (Cambridge University Press, Cambridge, 2011).

[4] I. Bloch, J. Dalibard, and W. Zwerger, Rev. Mod. Phys. 80, 885 (2008).

[5] M. R. Moldover, J. V. Sengers, R. W. Gammon, and R. J. Hocken, Rev. Mod. Phys. 51, 79 (1979).

[6] K. Damle, T. Senthil, S. N. Majumdar, and S. Sachdev, Europhys. Lett. 36, 7 (1996).

[7] S. Wessel, F. Alet, M. Troyer, and G. G. Batrouni, Phys. Rev. A 70, 053615 (2004).

[8] M. Rigol and A. Muramatsu, Phys. Rev. A 70, 031603 (2004); 72, 013604 (2005).

[9] S. Fölling, A. Widera, T. Müller, F. Gerbier, and I. Bloch, Phys. Rev. Lett. 97, 060403 (2006).

[10] Q. Niu, I. Carusotto, and A. B. Kuklov, Phys. Rev. A 73, 053604 (2006).

[11] D. Belitz, T. R. Kirkpatrick, and R. Saha, Phys. Rev. Lett. 99, 147203 (2007).

[12] T. Platini, D. Karevski, and L. Turban, J. Phys A 40, 1467 (2007).

[13] R. B. Diener, Q. Zhou, H. Zhai, and T. L. Ho, Phys. Rev. Lett. 98, 180404 (2007).

[14] T. Donner, S. Ritter, T. Bourdel, A. Öttl, M. Köhl, and T. Esslinger, Science 315, 1556 (2007).

[15] M. Holzmann and W. Krauth, Phys. Rev. Lett. 100, 190402 (2008).

[16] N. Gemelke, X. Zhang, C.-L. Hung, and C. Chin, Nature 460, 995 (2009).

[17] A. Bezett and P. B. Blakie, Phys. Rev. A 79, 033611 (2009).

[18] E. Taylor, Phys. Rev. A 80, 023612 (2009).

[19] M. Campostrini and E. Vicari, Phys. Rev. Lett. 102, 240601 (2009); 103, 269901(E) (2009).

[20] R. N. Bisset, M. J. Davis, T. P. Simula, and P. B. Blakie, Phys. Rev. A 79, 033626 (2009).

[21] Q. Zhou, Y. Kato, N. Kawashima, and N. Trivedi, Phys. Rev. Lett. 103, 085701 (2009).

[22] M. Rigol, G. G. Batrouni, V. G. Rousseau, and R. T. Scalettar, Phys. Rev. A 79, 053605 (2009).

[23] I. Hen and M. Rigol, Phys. Rev. A 82, 043634 (2010).

[24] S. Trotzky, L. Pollet, F. Gerbier, U. Schnorrberger, I. Bloch, N. V. Prokofev, B. Svistunov, and M. Troyer, Nat. Phys. 6, 998 (2010).

[25] M. Campostrini and E. Vicari, Phys. Rev. A 81, 023606 (2010).

[26] Q. Zhou and T.-L. Ho, Phys. Rev. Lett. 105, 245702 (2010).

[27] T.-L. Ho and Q. Zhou, Nat. Phys. 6, 131 (2010).

[28] L. Pollet, N. V. Prokof'ev, and B. V. Svistunov, Phys. Rev. Lett. 104, 245705 (2010).
[29] L. Pollet, N. V. Prokof'ev, and B. V. Svistunov, Phys. Rev. Lett. 105, 199601 (2010).

[30] S. Nascimbene, N. Nayon, F. Chevy, and C. Salomon, New J. Phys. 12, 103026 (2010)

[31] Q. Zhou, Y. Kato, N. Kawashima, and N. Trivedi, Phys. Rev. Lett. 105, 199602 (2010).

[32] M. Campostrini and E. Vicari, Phys. Rev. A 81, 063614 (2010); 82, 063636 (2010); J. Stat. Mech. (2010) P08020; (2010) E04001.

[33] S. L. A. de Queiroz, R. R. dos Santos, and R. B. Stinchcombe, Phys. Rev. E 81, 051122 (2010).

[34] S. Fang, C-M. Chung, P-N. Ma, P. Chen, and D-W. Wang, Phys. Rev. A 83, 031605(R) (2011).

[35] X. Zhang, C.-L. Hung, S.-K. Tung, N. Gemelke, and C. Chin, New J. Phys. 13, 045011 (2011).

[36] F. Crecchi and E. Vicari, Phys. Rev. A 83, 035602 (2011).

[37] K. W. Mahmud, E. N. Duchon, Y. Kato, N. Kawashima, R. T. Scalettar, and N. Trivedi, Phys. Rev. B 84, 054302 (2011).

[38] K. R. A. Hazzard and E. J. Mueller, Phys. Rev. A 84, 013604 (2011).

[39] G. Ceccarelli, C. Torrero, and E. Vicari, Phys. Rev. A 85, 023616 (2012); Phys. Rev. B 87, 024513 (2013).

[40] L. Pollet, Rep. Prog. Phys. 75, 094501 (2012).

[41] G. Ceccarelli and C. Torrero, Phys. Rev. A 85, 053637 (2012).

[42] Y. Khorramzadeh, Fei Lin, and V. W. Scarola, Phys. Rev. A 85, 043610 (2012)

[43] J. Carrasquilla and M. Rigol, Phys. Rev. A 86, 043629 (2012).

[44] G. Ceccarelli, J. Nespolo, A. Pelissetto, and E. Vicari, Phys. Rev. B 88, 024517 (2013).

[45] G. Ceccarelli and J. Nespolo, Phys. Rev. B 89, 054504 (2014).

[46] C. Bonati, M. D’Elia, and E. Vicari, Phys. Rev. E 89, 062132 (2014).

[47] M. E. Fisher, M. N. Barber, and D. Jasnow, Phys. Rev. A 8, 1111 (1973)

[48] J. Cardy, Finite-Size Scaling (North Holland, Amsterdam, 1988).

[49] S. L. Sondhi, S. M. Girvin, J. P. Carini, and D. Shahar, Rev. Mod. Phys. 69, 315 (1997).

[50] M. Campostrini, A. Pelissetto, and E. Vicari, Phys. Rev. B 89, 094516 (2014).

[51] V. Piazza, V. Pellegrini, F. Beltram, W. Wegscheider, T. Jungwirth, and A. H. MacDonald, Nature 402, 638 (1999).

[52] T. Vojta, D. Belitz, T. R. Kirkpatrick, and R. Narayanan, Ann. Phys. (Leipzig) 8, 593 (1999).

[53] M. Uhlarz, C. Pfleiderer, and S. M. Hayden, Phys. Rev. Lett. 93, 256404 (2004)

[54] C. Pfleiderer, J. Phys.: Cond. Matter 17, S987 (2005).

[55] W. Knafo, S. Raymond, P. Lejay, and J. Flouquet, Nat. Phys. 5, 753 (2009). 
[56] E. Jeckelmann, Phys. Rev. Lett. 89, 236401 (2002).

[57] A. Kuklov, N. Prokof'ev, and B. Svistunov, Phys. Rev. Lett. 92, 050402 (2004).

[58] G. G. Batrouni, V. G. Rousseau, and R. T. Scalettar, Phys. Rev. Lett. 102, 140402 (2009).

[59] J. Radic, A. Di Ciolo, K. Sun, and V. Galitski, Phys. Rev. Lett. 109, 085303 (2012)

[60] D. Yamamoto, T. Ozaki, C. A. R. Sá de Melo, and I. Danshita, Phys Rev. A 88, 033624 (2013).

[61] S. Peotta, L. Mazza, E. Vicari, M. Polini, R. Fazio, and D. Rossini, J. Stat. Mech. (2014) P09005.

[62] M. Piraud, Z. Cai, I. P. McCulloch, and U. Schollwöck, Phys. Rev. A 89, 063618 (2014)

[63] U. Schollwöck, Rev. Mod. Phys. 77, 259 (2005).

[64] M. Campostrini, J. Nespolo, A. Pelissetto, and E. Vicari, Phys. Rev. Lett. 113, 070402 (2014).

[65] M. Campostrini, J. Nespolo, A. Pelissetto, and E. Vicari, arXiv: 1410.8662.

[66] P. Pfeuty, Ann. Phys. 57, 79 (1970).

[67] S. Giorgini, L. P. Pitaevskii, and S. Stringari, Rev. Mod. Phys. 80, 1215 (2008).

[68] T. Esslinger, Ann. Rev. Cond. Mat. Phys. 1, 129 (2010).

[69] X.-W. Guan, M. T. Batchelor, and C. Lee, Rev. Mod. Phys. 85, 1633 (2013).

[70] A. Angelone, M. Campostrini, and E. Vicari, Phys. Rev. A 89, 023635 (2014).

[71] R. B. Potts, Math. Proc. Camb. Phil. Soc. 48, 106 (1952)

[72] F. Y. Wu, Rev. Mod. Phys. 54, 235 (1982).
[73] R. J. Baxter, J. Phys. C: Solid State Phys. 6, L445 (1973); R. J. Baxter, H. N. V. Temperley, and S. E. Ashley, Proc. R. Soc. Lond. A 538, 535 (1978).

[74] J. Sólyom and P. Pfeuty, Phys. Rev. B 24, 218 (1981).

[75] F. Iglói and J. Sólyom, J. Phys. C: Solid State Phys. 16, 2833 (1983).

[76] D. Kim, Phys. Lett. A 87, 127 (1981).

[77] R. J. Baxter, J. Phys. A 15, 3329 (1982).

[78] We thank one of the referees for pointing out this exact result.

[79] B. Nienhuis and M. Nauenberg, Phys. Rev. Lett. 35, 477 (1975).

[80] M. E. Fisher and A. N. Berker, Phys. Rev. B 26, 2507 (1982)

[81] V. Privman and M. E. Fisher, J. Stat. Phys. 33, 385 (1983).

[82] M. E. Fisher and V. Privman, Phys. Rev. B 32, 447 (1985).

[83] M. S. S. Challa, D. P. Landau, and K. Binder, Phys. Rev. B 34, 1841 (1986).

[84] V. Privman ed., Finite Size Scaling and Numerical Simulation of Statistical Systems (World Scientific, Singapore, 1990).

[85] J. Lee and J. M. Kosterlitz, Phys. Rev. B 43, 3265 (1991).

[86] C. Borgs and R. Kotecký, Phys. Rev. Lett. 68, 1734 (1992).

[87] A. Billoire, T. Neuhaus, and B. A. Berg, Nucl. Phys. B 396, 779 (1993).

[88] K. Vollmayr, J. D. Reger, M. Scheucher, and K. Binder, Z. Phys. B 91, 113 (1993).

[89] F. Iglói and E. Carlon, Phys. Rev. B 59, 3783 (1999).

[90] P. Calabrese, P. Parruccini, A. Pelissetto, and E. Vicari, Phys. Rev. B 70, 174439 (2004).

[91] G. G. Cabrera and R. Jullien, Phys. Rev. B 35, 7062 (1987).

[92] M. Campostrini, A. Pelissetto, and E. Vicari, arXiv:1501.03265. 